\title{
Libertà, necessità e storia. Percorsi dell'Esprit des lois di Montesquieu, a cura di Domenico Felice
}

\section{Gianmaria Zamagni}

\section{(2) OpenEdition}

12 Journals

\section{Edizione digitale}

URL: http://journals.openedition.org/studifrancesi/36172

DOI: 10.4000/studifrancesi.36172

ISSN: 2421-5856

\section{Editore}

Rosenberg \& Sellier

\section{Edizione cartacea}

Data di pubblicazione: 1 juillet 2005

Paginazione: 164-165

ISSN: 0039-2944

\section{Notizia bibliografica digitale}

Gianmaria Zamagni, «Libertà, necessità e storia. Percorsi dell'Esprit des lois di Montesquieu, a cura di Domenico Felice», Studi Francesi [Online], 145 (XLIX | I) | 2005, online dal 30 novembre 2015, consultato il 19 avril 2021. URL: http://journals.openedition.org/studifrancesi/36172 ; DOI: https:// doi.org/10.4000/studifrancesi.36172

\section{Questo documento è stato generato automaticamente il 19 avril 2021.}

\section{(c)}

Studi Francesi è distribuita con Licenza Creative Commons Attribuzione - Non commerciale - Non opere derivate 4.0 Internazionale. 


\title{
Libertà, necessità e storia. Percorsi dell'Esprit des lois di Montesquieu, a cura di Domenico Felice
}

\author{
Gianmaria Zamagni
}

\section{NOTIZIA}

Libertà, necessità e storia. Percorsi dell'Esprit des lois di Montesquieu, a cura di Domenico FELICE, Napoli, Bibliopolis, 2003, pp. 332.

1 Il volume, da Domenico Felice progettato e curato con la perizia e la precisione a cui ci ha oramai abituato, fa seguito al precedente Leggere l'«Esprit des Lois». Stato, società $e$ storia nel pensiero di Montesquieu, Napoli, Liguori, 1998, sempre a sua cura. E ad ideale continuazione di quello studio, anche Libertà, necessità e storia è costituito di sei contributi che ripercorrono la sestuplice partizione dell'opus magnum montesquieuiano. La Premessa del curatore, oltre ad illustrare brevemente i saggi, è utilissima per la ricca bibliografia, che vi si trova, delle opere sul Président pubblicate negli ultimi cinque anni in Francia, Stati Uniti, Svizzera, Germania e Italia.

In apertura di volume l'ampio saggio di Thomas CASADEI, Modelli repubblicani nell'«Esprit des lois». Un 'ponte' tra passato e futuro (pp. 13-74), offre, prima di ripercorrere il testo per analizzarvi diverse forme repubblicane, una «messa a punto concettuale» dei terminichiave della trattazione montesquieuiana delle forme di governo, della loro natura, del loro principio. In seguito, vengono mostrati, con Atene e Sparta da un lato, con Roma dall'altro (questo, definito «parfait»), due diversi «modelli» di repubbliche nel passato. Questi modelli vengono però complicati, nell'analisi dell'Esprit des lois, da due fattori cruciali: quello economico (detta problematicamente: il commercio di lusso può intaccare il principio della repubblica) e quello politico, esaminato nel caso della repubblica di Venezia (il potere non essendovi effettivamente diviso). Nei capitoli successivi Casadei va, con Montesquieu, al di là di Montesquieu, allargando la questione 
alla repubblica statunitense (qui, l'interpretazione montesquieuiana cui è data maggior attenzione, è quella di Thomas Paine). Nella parte finale, infatti, Casadei giunge a interrogarsi, in direzione affatto normativa, su una forma di repubblicanesimo che possa essere «espressivista» (per dirla con Charles Taylor) o «pluralista», e non «imperiale».

Il curatore Domenico Felice, nel suo Autonomia della giustizia e filosofia della pena nell'«Esprit des lois» (pp. 75-136), si occupa invece di giustizia e filosofia penale. Nei libri IX-XIII, seconda parte dell'Esprit des lois, cui corrisponde idealmente il saggio, il potere giudiziario viene consacrato a potere fondamentale della Stato, ed assume il carattere di vero e proprio «fattore discriminante tra governo moderato e dispotismo». L'organizzazione del giudiziario viene in seguito analizzata nei modelli francese e inglese di monarchia, "due modelli, diversi e antitetici, di organizzazione del «terribile» potere di giudicare» (p. 104). La seconda parte del contributo viene da Felice poi dedicata all'analisi della filosofia penale dell'Esprit des lois. E anche in questo campo, il capolavoro del Président à mortier pare uno spartiacque nel suo secolo, autentico momento di fondazione della giurisprudenza liberale, a salvaguardia di libertà $\mathrm{e}$ sicurezza del cittadino e della proprietà.

Carlo BORGHERO, nel saggio Libertà e necessità: clima ed 'esprit général'nell' «Esprit des lois» (pp. 137-202), pone in apertura l'attenzione alla Défense pubblicata da Montesquieu nel 1750, ove il Président mette al riparo le proprie tesi sul clima dagli attacchi dei moralisti al suo Esprit des lois. Critiche importanti su questo aspetto gli vennero mosse del resto da Voltaire e da d'Alembert. Ma Borghero non manca di dire che non vi è in Montesquieu alcun determinismo climatico, come si è voluto troppo spesso ravvisare, quanto un «modello epistemologico organicistico» (p. 153). L'esprit di un popolo, di una nazione, che l'autore del saggio fa risalire nell'elaborazione del Président fino al perduto De la différence des génies (1717), è condizionato tanto da cause fisiche, quanto da cause morali, e sono queste ultime ad avere la meglio, e possono dunque correggere le prime. Con la lezione del caso romano, Montesquieu dimostra la tesi secondo cui «il luogo del significato della storia è dunque l'esprit général», non meramente "l'hasard d'une bataille» (pp. 158 s.). È dunque sulla scorta della profonda conoscenza di quell'organismo, che il legislatore deve governare: «nella capacità di cogliere esattamente i rapports tra le leggi e l'esprit général di una nazione si misura la [sua] saggezza» (p. 199).

5 Il saggio Demografia, economia e società in Montesquieu (pp. 203-242), è la ristampa di due contributi di Salvatore RoTTA; si intende così rendere omaggio all'insigne studioso di Montesquieu, di recente scomparso. Rotta, con erudizione immensa, ricostruisce l'intero clima di discussione sul tema demografico al tempo di Montesquieu, ma di quest'ultimo biasima le fonti. Esse facevano sì che Montesquieu, soprattutto nelle Lettres persanes ritenesse la popolazione mondiale in forte calo. Nell'Esprit des lois, «pur tenendo fermo alla tesi della tendenza generale allo spopolamento, egli è molto più moderato nelle sue formulazioni» (p. 213). Il contributo poi si occupa di aspetti economici legati alla demografia: lo sviluppo dell'agricoltura e del commercio, il lusso, la tassazione e i connessi problemi di uguaglianza o disuguaglianza sociale. La legge generale, con cui Rotta conclude citando il Président, è che «i paesi non sono coltivati in favore della loro fertilità, ma della loro libertà» $(E L$, XVIII, 3).

6 Il lucido contributo di Lorenzo BIANCHI, Leggi divine e leggi umane. Note sulla religione nel libro XXVI dell'«Esprit des lois» (pp. 243-276), ha in oggetto il libro XXVI, il meno trattato 
quando si è scritto di religione nell'Esprit des lois, di cui si mostra nel dettaglio il contenuto, ma del quale non di meno si sottolinea l'importanza teorica. Questo libro, infatti, si premura di dipanare casi di confusione, sovrapposizione, conflitto, tra le sfere religiosa e civile. Casi di particolare rilevanza sono il matrimonio, cui sono dedicate diverse pagine (254-257 e 267-271), e il tribunale dell'Inquisizione (259-267, in cui sono trattate anche le critiche dei teologi Concina e Bottari). Con questo intento di conferire ordine alla giurisprudenza su tali controverse materie, il libro XXVI appare in tutta la sua valenza teorica generale, «una sorta di modello normativo capace di fornire al legislatore indicazioni pratiche e ideali» (p. 273). In conclusione, Bianchi mostra quanto lo stesso Cesare Beccaria avesse ben presente, per questo stesso motivo, le istanze presenti in questo testo.

7 Il saggio di Umberto ROBERTO, Montesquieu, i Germani e l'identità politica europea (pp. 277-322), in conclusione, è dedicato della sesta parte dell'Esprit des lois, luogo in cui il Président rilegge i classici sulla Germania di Cesare, ma soprattutto di Tacito, e li confronta con i codici delle leggi di questi popoli. Questi dicono di quella storia, e quella storia dice di questi codici. Le loro leggi, scrive Roberto, «appaiono sottese da un comune esprit, da un fondo culturale che rimanda con suggestiva precisione alla cultura descritta da Tacito e da Cesare» (p. 282). Del canto suo, Montesquieu realizza dunque in queste pagine una compiuta storia politica, attraverso l'attento studio delle istituzioni dell'Europa in alcuni importanti momenti chiave: ascesa e declino di Roma, invasione dei popoli germanici, dei Franchi e la loro vitalità prorompente, il feudalesimo, e la nascita degli Stati moderni. Ma un'importanza cruciale ha la libertas germanica, «svolta culturale profonda» (p. 300), tanto che il capolavoro stesso della legislazione, rappresentato dalla costituzione inglese «questo bel système [,] è stato trovato nelle foreste» (p. 320). Il volume edito da Bibliopolis - di cui è da lodare l'eleganza grafica ed editoriale - è corredato anche dall'indice dei nomi. 